\title{
Die Kirche und die Medien: zum Multi-Media-Gebrauch
}

\author{
von G. Ralph Milton
}

In den letzten Jahren hat man dem Wort Medium ein neues Präfix verliehen. Kommunikatoren sprechen von "Multi-Media", einem Wort, das schon zu einem Modewort wurde, das aber auch ein Faktum bezeichnet. - Als Modewort wird MultiMedia von solchen gebraucht, die, abgesehen davon, daß „alle es benutzen“ und daß es deswegen schon richtig sein muß, nur eine unklare Vorstellung von diesem Ausdruck haben.

Tatsächlich existiert die Multi-Media-Kommunikation des Menschen schon, seitdem der Höhlenmensch seine Erzählung mit Gesten unterstrich. Mit anderen Worten: Multi-Media bezeichnet grundsätzlich die Benutzung von zwei oder mehr Kommunikationskanälen für ein einziges Ziel. Ein düsterer Blick etwa, ein Schimpfwort und ein schneller Schlag auf die Nase könnten genau als eine effektive Multi-MediaKommunikation bezeichnet werden. Auch ein Gottesdienst könnte wegen des Gebrauchs von religiösen Symbolen, der Architektur, der Musik, der liturgischen Handlung, ja selbst wegen des Weihrauchgeruchs als Multi-Media-Kommunikation bezeichnet werden, bei der all diese Dinge eine gemeinsame Kommunikationserfahrung darstellen, die wir Gottesdienst nennen.

Häufiger denkt man allerdings bei Multi-Media an eine Vorführungsart, bei der eine Reihe von Filmprojektoren gebraucht werden, die ihre Bilder auf mehrere Bildschirme werfen, wozu dann vielleicht noch farbige Beleuchtung, Tonband, Luftballons, bestimmte Gerüche und wirkliche Schauspieler kommen. Es ist der McLuhaneske Stil, für den die Expo in Montreal 1967 das lebendigste Beispiel wurde. Tatsächlich ist der Begriff sehr weit und schließt alles mögliche ein.

Hier geht es darum, in einem kurzen Uberblick einige Informationen über MultiMedia-Gebrauch im wissenschaftlichen und werblichen Bereich zu geben und $\mathrm{zu}$ versuchen, die Bedeutung dieser Studien für die kirchliche Kommunikation deutlich $\mathrm{zu}$ machen. Auch soll auf einige Untersuchungen hingewiesen werden, die helfen können, die effektivste Aufgabe der einzelnen Medien im Rahmen einer totalen Kommunikation, der die Kirchen sich gegenübersehen, näher zu bestimmen.

Der Ausdruck Multi-Media wird im allgemeinen in der Publizistik für den systematischen Gebrauch verschiedener Medien innerhalb eines sorgfältigen Erziehungs- oder

G. Ralph Milton, früherer Direktor für Hörfunk und Fernsehen der Massenmedienkommission des National Council of Churches der Philippinen, ist "Director of Operational Research" bei RAVEMCCO (Radio, Visual Education and Mass Communications Committee of the National Council of the Churches of Christ), New York. Er ist Verfasser eines in Zusammenarbeit mit der World Assocation for Christian Broadcasters herausgegebenen Handbuches für Hörfunk-Programmarbeit in Entwicklungsländern (vgl. CS 2:1969, S. 182). - Die Herausgeber sehen in diesem Beitrag einen ersten Ansatz zum MultiMedia-Verständnis skizziert wie ihn der Kommunikations-Experte eines großen kirchlichen Zusammenschlusses in den USA begreift. Vertiefende Uberlegungen zum Thema stehen in CS noch an. 
Werbeprogramms gebraucht. Die verschiedenen Medien werden auf ihre besonderen Stärken, Schwächen und inhärenten Charakteristika analysiert und einzeln oder in verschiedenen Kombinationen gebraucht, um jene Gesamtaufgabe zu erreichen, für die sie am besten geeignet sind. Es geht um eine Medienplanung, die vollständig innerhalb des Gesamtprogramms koordiniert ist, sei es für den Rechenunterricht von Schulkindern, für eine Wahlkampagne, für die Entwicklung eines Agrarprojektes in Entwicklungsländern oder für die evangelistische Ausbreitung einer Kirche. ${ }^{1}$

Leider glaubt man in manchen Kreisen, man würde automatisch zu einem effektiven Multi-Media-Gebrauch kommen, wenn man einfach Presse, Rundfunk und Film unter einem Management zusammenfaßt. Andere scheinen zu glauben, daß die einfache Uberflutung des Empfängers mit Mitteilungen durch jedes mögliche Medium zur wirksamsten Kommunikation führen müsse. Beide Versuche können produktiv sein oder auch völlig nutzlos. Leider sind in den Kirchen beide "Erfolgs"-Varianten festzustellen.

Ein flüchtiger Blick in die Werbeliteratur zeigt, daß man dem "systematischen Zugang " vertraut, bei dem die Planung für den Mediengebrauch ein integraler Bestandteil des gesamten Unternehmens ist. Dieser Zugang bezeichnet eine Managementtechnik (manchmal als "Program Evaluation“, als "Review Technic" oder als "Critical Path Method"), die dazu bestimmt ist, eine "Zeit-Ereignis"-Analyse des Projektes zu entwickeln. Bei diesem System werden Werbe-Experten schon in die Vorplanung der Entwicklung eines Produktes einbezogen. Die Produzenten kommen nicht zum Werbefachmann und sagen "Hier, verkaufen sie dies“, weil sie der Überzeugung sind, daß ein Produkt als solches schon kommunikativ sei und eine Chance habe. Von vornherein werden die Verpackung, die Werbung und der Verkauf für jedes Produkt sorgfältig aufeinander abgestimmt, so daß alle sich gegenseitig ergänzen.

Die Werbewirtschaft hat auf diesem Gebiet eine intensive Forschung betrieben und offensichtlich brauchbare Methoden gefunden. Schon die flüchtige Prüfung einiger alltäglicher Produkte gibt dafür eine reichliche Evidenz. Leider sind diese Forschungen im einzelnen nicht einsehbar. Wegen der starken Konkurrenz der beteiligten Firmen werden die Ergebnisse solcher Untersuchungen sorgfältig unter Verschluß gehalten.

Ford hat beispielsweise eine Untersuchung machen lassen, die über eine Million Dollar kostete, um die vier verschiedenen Medien Hörfunk, Presse, Fernsehen und Plakat auf ihre Werbe-Wirksamkeit beim Autoverkauf zu testen. J. B. Haskins berichtet von dieser Forschung und kommentiert: "Wie bei den meisten Werbeuntersuchungen dieser Art wurden die Ergebnisse nicht veröffentlicht. “2 Nimmt man an, daß die Firma ihre Werbepolitik nach diesen Forschungsergebnissen ausrichtete, dann müssen sie gezeigt haben, daß ein koordinierter Multi-Media Approach sich als besonders effektiv erwies; denn Ford hat genau diese Werbeart seit jener Zeit angewandt.

Die amerikanische Telephon- und Telegrafengesellschaft (ATT) erstellte einen Bericht über die Ergebnisse einer Studie in 16 Städten, in denen Zeitungen und Fernsehen in verschiedenen Kombinationen gemeinsam für ein bestimmtes Kommunikationsziel eingesetzt wurden. Robert Cunningham, der leitende Statistiker der ATT, gab als ein Ergebnis bekannt, daß die Kombination eines großen Fernsehgebrauchs mit niedrigem Zeitungsgebrauch fast den gleichen Bewußtseinsgrad erzielt habe wie die Kombination eines hohen Zeitungsgebrauchs mit starker Fernsehwerbung. Wo aber 
die Fernsehbenutzung niedrig war, konnte ein hoher Kostenaufwand für Zeitungen dies nicht kompensieren."

Eine äußerst aufschlußreiche, nicht-werbliche Studie ist die Feldforschung von Spector, Torres, Lichtenstein und Preston. Die Agentur für Internationale Entwicklung ließ die vergleichbaren Wirkungen im Gebrauch von drei verschiedenen Medien bei der Einführung von Neuerungen in entlegenen Anden-Städten Ekuadors untersuchen.

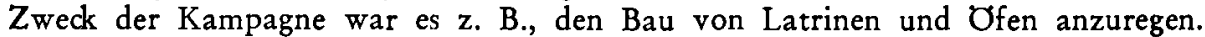
Der Bericht zeigt, daß sich Hörfunk und audio-visuelle Mittel beide als gleich wirksam erwiesen in der Werbung für die Teilnahme, daß sie aber differierende Funktionen ausübten bezüglich der verschiedenen Arten der Aktion und des investierten Aufwands. ${ }^{4}$

Das US-Landwirtschaftsministerium kam bereits bei 1955 durchgeführten Studien zu der Schlußfolgerung, daß der gemeinsame Einfluß verschiedener Methoden (Medien) im Regelfall notwendig sei, um eine gewünschte Änderung eingeübter Praktiken zu erreichen. ${ }^{5}$

Eine Untersuchung an mehr als 1100 Haushaltsvorständen in fünf Staaten ergab, daß ungefähr 46 Prozent jener, die einer Werbung durch ein oder zwei Medien ausgesetzt waren, sich bewegen ließen, ihre Gewohnheiten zu ändern; von jenen aber, die drei oder vier Medien ausgesetzt waren, wurden 68 Prozent zu einer Anderung veranlaßt; bei acht oder mehr medialen Anwendungsformen stieg diese Rate dann sogar bis zu 97 Prozent. ${ }^{\circ}$

Eine der Schwierigkeiten, die sich für die Bewertung der Inter-Media-Experimente ergeben, liegt in der ungeheuren Verschiedenheit der jeweiligen Mittel und Methoden. Eine Vergleichsstudie der Illustrierten "Look" fand nur wenige Unterschiede $z$ wischen Fernsehen und Presse, wenn es um die eigentliche Information ging. Nach dem Vizepräsidenten der Zeitschrift, Joel Harnett, erwies sie, daß Presse und Fernsehen sich gegenseitig "ergänzen und unterstützen“. Als bedeutendstes Ergebnis zeigte sich jedoch, daß eingängige Anzeigen mit gutem Design in beiden Medien wirkungsvoll waren, während schwache Anzeigen in beiden Medien gleichermaßen schlecht ankamen. ${ }^{7}$

Ohne Zweifel müssen zur Frage der Multi-Media noch viele Forschungsdaten freigelegt und noch mehr müßte speziell im Bereich der kirchlichen Kommunikation und in den Entwicklungsländern der Welt in dieser Richtung getan werden. Solange dies nicht der Fall ist, wird sich die kirchliche Publizistik ständig erneut der Notwendigkeit gegenübersehen, für jeden einzelnen Fall die wirksamsten Kanäle zu wählen. Deswegen scheint es angebracht, einige Studien über Medienvergleiche und verwandte Gebiete anzuführen.

Welches Medium ist am besten zu gebrauchen? Die Kirchen haben viele Vorfragen zu klären, bevor sie darauf eine Antwort geben können. Die eigentliche Frage müßte lauten: Wie kann man das Medium am besten gebrauchen? Was soll vermittelt werden? An wen? Warum? Jede dieser Fragen impliziert eine Fülle von Problemen, die zu beachten sind, will man die übergeordneten Fragen klären. Berücksichtige man alle Studien, schreibt Hower Hsia, dann müsse man zugeben, „daß viele Faktoren die relative Wirksamkeit der verschiedenen Kommunikationsformen beeinflussen und daß keines der Medien an sich einem anderen überlegen ist $^{\text {" }} .8$ Eine solche Warnung sollte vor allem von Kirchenmännern ernst genommen werden, die in der Versuchung sind, ihr "Lieblings"-Medium zu fördern.

Welches sind nun Faktoren, die die relative Wirksamkeit der verschiedenen Medien beeinflussen? Diesbezügliche Untersuchungen wurden - speziell im Bereich der 
Erziehung - noch nicht abgeschlossen. Die bisher vorliegenden Ergebnisse reichen nicht aus, um sichere Schlußfolgerungen $\mathrm{zu}$ ziehen. Einige Grundlinien scheinen sich allerdings abzuzeichnen. Sie betreffen: a) Publikumszusammensetzung und $\mathrm{Me}$ diengebrauch, b) psychologische Reaktionen gegenüber den Medien. - Für die zweite Kategorie gibt Hsia eine brauchbare Liste von Charakteristiken für Hör- und Sehmedien, wenn er zusammenfaßt: „Im allgemeinen haben Darbietungen mit Bild den Vorteil größerer Dimension mit mehr Eindrücken und Anhaltspunkten, sind dadurch allerdings auch nicht so klar und eindeutig wie das gedruckte Wort." - "Das Problem der Wirksamkeit der Kommunikation“, schreibt er, „besteht darin, das eine Medium durch das andere so $\mathrm{zu}$ unterstïtzen, daß gegenseitige Störungen möglichst ausgeschaltet werden. “9

Dem Verlangen nach dem qualitativen Maßstab für einen Media-Vergleich kann eine Studie dienen, die vom Fernsehbüro für Werbung in den USA angestellt wurde. ${ }^{10}$ Die Studie fragte, warum die Menschen jedes Medium gebrauchen und welche Vorteile sie daraus ziehen. Die persönlichen Vorteile aus dem Mediengebrauch so ging aus der Studie hervor - waren Information und sieben verschiedene Arten emotionaler Beteiligung: 1. stellvertretende Erfahrungen, 2. Neugierde und Klatsch, 3. Belustigung, 4. ästhetischer Genuß, 5. Selbstbestätigung, 6. menschlicher Kontakt und 7. Freundschaft. Die Untersuchung zeigte, daß nicht alle Rezipienten beim Gebrauch der Medien auf jede dieser Erfahrungen aus sind und daß gewisse Medien bestimmte Effekte besser erzielen als andere. Bei Zeitungen scheinen die bedeutendsten gesuchten Werte Neugierde und Klatsch (Einzelheiten über Menschen und bekannte Persönlichkeiten), die Belustigung und der Reiz zur Selbstbestätigung (Rätsel!) zu sein. Bei Magazinen scheinen nachempfundene Erfahrungen (Geschichten!), Klatsch, Humor und ästhetischer Genuß im Vordergrund zu stehen. Den besonderen Wert des Hörfunks scheinen die Befragten im ästhetischen Genuß (Musik) zu sehen sowie in mitmenschlichem Kontakt und freundschaftlichen Empfindungen, wenn der Hörer alleinstehend ist. Beim Fernsehen fühlen sich die Befragten zu einem guten Teil von allen sieben der aufgezeigten Arten angesprochen. Das Fernsehen ist auch nicht nur das Medium mit den meisten, sondern auch mit den tiefgreifendsten Emotionsmöglichkeiten. Der Hinweis, daß diese Studie vom Büro für Fernsehwerbung durchgeführt wurde, kann allerdings die Ergebnisse relativieren.

Nützlicher sind wohl Studien über die Zusammensetzung des Publikums und den Mediengebrauch. Untersuchungen, die in Kolumbien, in Chile und in den Vereinigten Staaten 11 angestellt wurden, zeigen, daß sich im allgemeinen das Publikum der verschiedenen Medien überschneidet, d. h. daß die Benutzung des einen Mediums einen positiven Einfluß auf den Gebrauch eines anderen Mediums hat. Vor einigen Jahren hat Lazarsfeld die Beziehungen zwischen Hörfunk und Presse untersucht. Er kam zu dem Schluß, daß der Hörfunk „die Lesegewohnheiten der Bevölkerung nicht beeinträchtigt hat" ${ }^{12}$ Dies ist eine der wenigen Studien, die gemacht wurden, als der Hörfunk noch das einzige weitreichende elektronische Kommunikationsmittel war. Natürlich gibt es aus neuerer Zeit Untersuchungen über die Beziehungen zwischen Fernsehbeteiligung und Presse. Eine Untersuchung über den Einfluß des Fernsehens auf Schulkinder in Großbritannien ergab, daß die Zahl der von Kindern in vier Wochen gelesenen Bücher nach der Einführung des Fernsehens leicht abnahm. Andererseits verbesserte das Fernsehen den Lesegeschmack der Kinder. ${ }^{13}$ Parker bestätigt neuere Forschungen, wenn er schließt, „daß die Bibliothekenbenutzung nach der Einführung des Fernsehens geringer ist als zur Zeit, da es kein Fernsehen gab, 
und daß ein Rückgang der Romanausleihe zu verzeichnen ist gegenüber einer $\mathbf{Z u}$ nahme der Sachbuchausleihe, wenn das Fernsehen ins Haus kommt “. ${ }^{14}$

Besonders hilfreich für die Kommunikations-Arbeit in Entwicklungsländern ist eine in Kolumbien durchgeführte Studie. Der Bericht beschreibt die Beziehung zwischen dem Media-Gebrauch und dem Analphabetentum, dem Einfühlungsvermögen, der Bereitschaft zu Neuerungen, zum politischen Wissen und den Leistungsmotiven. Man könnte annehmen, daß Landarbeiter, solange sie nicht lesen können, wenig durch die Presse beeinflußt werden, es sei denn durch Mittelsmänner. Interessanterweise aber zeigt die Studie deutlich, daß gerade Landarbeiter, die lesen können, viel häufiger auch Radiohörer sind als Analphabeten. Hörfunk und Fernsehen sind wesentlich stärker der Alphabetisierung zugeordnet als der Film. Dies mag allerdings auch durch die Wechselwirkung zwischen Alphabetisierung und wirtschaftlicher Lage begründet sein; denn viele Analphabeten sind eben zu arm, um sich ein Radio oder einen Fernsehempfänger kaufen zu können, während der Filmbesuch im ländlichen Kolumbien entweder kostenlos oder doch sehr billig ist. „Funktionale Elementarbildung befähigt die Landarbeiter in einer sich entwickelnden Gesellschaft zum Gebrauch der Massenmedien; dies wiederum führt zu verschiedenen Modernisierungsfolgerungen, wie Bereitschaft für Neuerungen, größeres Verständnis, politisches Wissen und wachsendes Bildungsverlangen. "15 Im Lichte dieser Erkenntnisse erscheint es nützlich, Entwicklung als einen ganzheitlichen Prozeß zu sehen, der auch die wirtschaftlichen, sozialen, geistigen und ethischen Bereiche umfaßt, die alle miteinander durch den Kommunikationsprozeß begriffen und entwickelt werden.

Eine neuere Studie über Mediengebrauch in den USA zeigt, daß verschiedene sozioökonomische Gruppen die Medien sehr verschieden gebrauchen. ${ }^{16}$ Obwohl diese Ergebnisse zu detailliert sind, um hier einzeln wiedergegeben werden zu können, darf man doch verallgemeinernd sagen, daß Personen mit höherer Bildung (weniger als ein Prozent der Gesamtbevölkerung) stark zur Presse hin tendieren, während solche mit einem geringen Bildungsgrad (50 bis 60 Prozent der Bevölkerung) die elektronischen Medien bevorzugen. Eine Ausnahme bildet die Nachrichtengebung. Darin gebrauchen beide Gruppen sowohl Zeitungen als auch Rundfunk sehr stark als Informationsquellen, auch wenn die Gruppe mit höherem Bildungsstand sich hierbei noch etwas mehr auf Zeitungen verläßt als die Gruppe mit dem geringen Bildungsstand. Die zwischen beiden Bildungsgruppen stehende Bevölkerungsschicht benutzt überwiegend den Hörfunk.

Es gibt nur wenige zugängliche Untersuchungen, um die Wirksamkeit des Mediengebrauchs auf lange Sicht zu bestimmen. Sie widmen sich zu oft nur einem einzelnen Film, einem einzelnen Programm, einer einzelnen Presseserie oder einem Gegenstand, der für das Publikum von nur geringem Interesse ist. Man weiß noch sehr wenig über den Einfluß der Massenmedien auf lange Sicht oder auch über deren Tiefgang. Vielleicht wird gerade aus diesem Grund den „prophetischen“ Aussagen eines Marshall McLuhan eine solche Aufmerksamkeit geschenkt.

Immerhin kann man auf eine Anzahl von Untersuchungen zurückgreifen, die $Z_{w}$ eifel an der Tatsache ausschließen, daß die Beziehungen eines Menschen zu Familie und Freunden sein Verhalten und seine Bildung mehr ändern als eines oder alle Massenmedien. Diese Feststellung wird mit fast monotoner Regelmäßigkeit getroffen. Sie gilt besonders für tiefer gehendes Verhalten und Meinen von der Art der Glaubensüberzeugungen. Aus dieser Sicht erscheinen die Massenmedien nicht als stärkste Waffe im geistigen Kampf um die Seele der Menschen. Es ist tatsächlich zweifelhaft, ob sie auch nur teilweise erfolgreich sein können ohne eine erneuerte 
und neu belebte Kirche. Sicher aber können sie nie Ersatz-Funktionen ausüben für die Arbeit einer Gemeinde und einer Gemeinschaft.

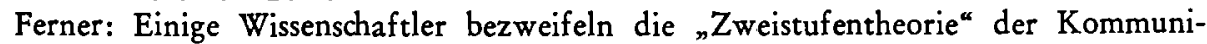
kation, bei der Ideen und Informationen von „wissenden “ Personen, die ihre Informationen von den Medien beziehen, an andere weniger informierte Personen weitergegeben werden. "Gerade in Bezug auf komplizierte und abstrakte Angelegenheiten, wie das Weltgeschehen... scheint die Information von einer Person zur anderen weitergeleitet zu werden, doch geschieht dies in mehreren Etappen. ${ }^{17}$ Andererseits aber haben Männer wie Apaton $\mathrm{Pal}$ und andere herausgefunden, daß die Zweistufentheorie größeren Wert in traditionellen Kulturen hat, wo die Meinungsbildner (opinion leaders) eine fest verwurzelte Funktion besitzen. ${ }^{18}$

Was immer auch das Endergebnis dieser Kontroverse sein wird, vor allem scheint klar zu sein, daß die Medien sich gegenseitig fördern, wenn sie mit persönlicher Kommunikation koordiniert werden. Diese Feststellung sollte von der Kirche sorgfältig registriert werden. Die UNESCO hat mit Experimenten bei der Frauenbildung im Senegal herausgefunden, daß Fernsehprogramme, wenn sie in Verbindung mit Diskussionsgruppen rezipiert wurden, wesentlich effektiver waren als im EinzelEmpfang. "Diskussionen, die dem Programm folgen, sind von entscheidender Bedeutung. Gerade während dieser Diskussionen zeigt sich die stimulierende und aktivierende Funktion des Bildungsfernsehens, das die Teilnehmer über den eigentlichen Unterrichtsstoff hinausführt." - "Diskussionsgruppen sind in Verbindung mit einem Fernsehbildungsprogramm notwendig, um die Informationen gesellschaftlich dynamisch zu machen." ${ }^{19}$ Der Erfolg von Radio-Foren in Kanada, Indien und in vielen anderen Teilen der Welt ist ein zusätzlicher Beweis.

Ein auf praktischem Gebiet vor zehn Jahren begonnenes Experiment zeigt noch heute die Ausführbarkeit einer Kombination von interpersonaler Kommunikation und Massenkommunikation. In der Umgebung von Dumagete City (Philippinen) wurden Entwicklungsexperten bei der Programmgestaltung einer protestantischen Hörfunkstation eingesetzt. Während ihrer Besuche bei den Bauern auf dem Felde trugen sie Tonbandgeräte mit sich. Jedesmal, wenn wichtige Probleme diskutiert wurden, nahm man sie auf Band und gebrauchte sie bei den Hörfunksendungen. Bauern, die mit neuen Methoden Erfolge erzielten, wurden öffentlich gelobt und so in ihrem Ansehen gehoben. Noch wichtiger aber war, daß die Landwirtschaftsfachleute die Möglichkeit hatten, die Bauern zu bestärken und jenen fast täglich Ermunterung und Instruktion zu vermitteln, die sie einige Monate vorher besucht hatten. Ein ähnliches Programm wird jetzt von Katholiken in Cagayan de Oro (Philippinen) durchgeführt.

Ein erfolgreiches Multi-Media-Experiment kirchlicher Publizistik wurde in Los Angeles in einem von Mexikanern und Amerikanern bewohnten Gebiet unternommen. Der Versuch zeigte, daß man Menschen mit Vertretern der Kirche in unmittelbaren Kontakt bringen kann, "wenn Dienstleistungen, die sie nötig haben, von der Kirche angeboten und wenn sie durch alle verfügbaren interpersonellen und medialen Kommunikationsmöglichkeiten bekannt gemacht werden ". ${ }^{20}$

Auch wenn diese Übersicht nur ein skizzenhafter Versuch sein kann, wird daraus doch für die Kirche genügend klar, daß sie sich bei allen Planungen ihres MedienProgramms über die Anwendung einer totalen Kommunikationsstrategie ernstlich Gedanken machen muß. Es wird eher die Regel als die Ausnahme sein, daß Einzelne oder Gruppen innerhalb der Kirchen darin fortfahren, dieses oder jenes favorisierte Medium, sei es Fernsehen, Zeitung oder Zeitschrift, zu fördern, als ob es jeweils 
in sich einen höheren Wert besäße. Wichtiger und wesentlicher ist es jedoch, alle Aktivitäten der Kirche auf pfarrlicher und nationaler Ebene als eine wesentliche Kommunikationsaufgabe zu sehen und alle Aspekte entsprechend zu berüdksichtigen und zu planen.

Am zukunftsträchtigsten aber ist die Erkenntnis, daß sich die Kirchen nicht mehr länger den Luxus leisten können, dieses oder jenes publizistische Mittel allein zu fördern. Wenn auch noch nicht alle Fragen des Multi-Media-Gebrauchs beantwortet sind, werden doch die Umrisse deutlich.

\section{Anmerkungen:}

1. Eine annotierte Bibliographie zur Multi-Media-Forschung findet sich im Grundlagendienst der Vierteljahrsschrift "Fernsehen und Bildung“, München 3:1969, Heft 4, Beilage S. III-XIII.

2. Jack B. Haskins: How to evaluate Mass Communications, New York 1968, S. 72.

3. Mündlicher Bericht vor der Association of National Advertisers Workshop, 1965.

4. Haskins: a.a.O., S. $56 \mathrm{f}$.

5. Wilson and Galleys (Hrsg.): Extension Service Circular 495 Washington D. C. (Department of Agriculture), 1955.

6. ebda.

7. - : Good ads score in all media-"look“, in: „Broadcasting“, Washington D. C., vol. 63, July 16, S. 30 .

8. Hower J. Hsia: On Channel Effectiveness, in: „AV Communication Review“, Washington D. C. $26: 1968$ (Nr. 3), S. 249.

9. ebda, S. 247.

10. E-Motion, TVB Presentation Highlights, Bulletin o. J. und ohne Datum.

11. Vgl. Everett M. Rogers und Johannes C. Van Es: Opinion Leadership in Traditional and Modern Columbian Communities, (Michigan State University, Department of Communications, Report 2), Michigan 1964. - Roy E. Carter Jr. und Orlando Sepúlveda: Some Patterns of Mass Media Use in Santiago de Chile, in: „Journalism Quarterly“, Iowa City, 4:1964, S. 216-224.

12. P. F. Lazarsfeld: Radio and The Printed Page, New York 1944, S. 329.

13. Hilde Himmelweit, A. N. Oppenheim und Pamela Vince: Television and the Child. An Empirical Study of the Effect of Television on the Young, London 1958, S. 486.

14. Edwin B. Parker: The Effects of Television, in: „Public Opinion Quarterly", New York, 27:1963, S. 585-587.

15. Everett M. Rogers: Mass Media Exposure and Modernization among Columbian Peasants, in: "Public Opinion Quarterly“, New York, 29:1965, S. 618-624; vgl. ferner: ders.: Modernization among Peasants, The Impact of Communication, New York 1969.

16. John P. Robinson und James W. Swinehart: World Affairs and the TV-Audience, in: "Television Quarterly", Syracuse/New York, 7:1968, S. 40-59, hier S. 47.

17. ebda, S. 54.

18. Aus persönlichen Gesprächen und der Kenntnis unveröffentlichter Unterlagen, die dem Vf. während seiner Zusammenarbeit mit Dr. Pal an der Silliman Universität in Dumagete (Philippinen) zugänglich waren.

19. Unesco (Hrsg.): Television and the social education of women, Reports and Papers on Mass Communication Nr. 50, Paris 1967, S. 9 und 34.

20. Perrow: A Strategy of Total Communication to Specific Cultural Groups, Part II, unveröffentlichter Bericht der United Presbyterian Church 1968, S. IV.

\section{S U M M A R Y}

Use of the term "multimedia" is a $\mathrm{fad}_{\text {ad }}$ when spoken by persons with only a dim understanding of the word; as a fact, man has been communicating through "multimedia" ever since the first caveman punctuated his narrative by waving his hands. How effective 
multimedia can be and how the Church could work in this way is shown in this summary of some relevant research done by various - especially industrial - agencies in the United States. (Full English text available from Rome editor CS.)

\section{RESUMEN}

El uso del término "multimedia" es el favorito de aquellas personas que apenas conocen su significado real; el hecho es que el hombre ha venido sirviéndose de "multimedia* desde que el primer hombre de las cavernas se servía de sus manos para acentuar lo que queria decir. Este resumen, realizado por diversas e importantes agencias de investigación, especialmente industriales, de los Estados Unidos, muestra en qué medida son efectivos los "multimedia“ y la forma en que deben ser utilizados. 